\section{Adaptive thinking as a heuristic in evolutionary psychology}

Recently, in evolutionary
psychology, many theorists have
employed adaptive thinking
as a heuristic as it generates
hypotheses that can later be
tested using the standard
scientific procedure. A heuristic
is a pragmatic method that,
while not being complete or
optimal, provides sufficient
approximate results for later use.
Professor Shunkichi Matsumoto
of Tokai University, Tokyo
focuses his research on the use
of heuristics in evolutionary
psychology, and in turn seeks
to clarify the role that adaptive
thinking has to play within the
discipline.

volutionary psychology is a scientific heuristics in evolutionary psychology - discipline that has had its fair Share of controversy regarding its
methodology and this does not appear changig any ins does Fo appear to there have been many theorists who have doubted adaptive thinking (a strategy of inferring evolutionary behaviours from valid method within the discipline in recent years however there has been a shift in research behaviours in justifying adaptive thinking as a good heuristic (a pragmatic method of providing sufficient approximate results, even if the method itself is not perfect or complete) in order to generate new and interesting hypotheses about the huma mind, that can later be put under the rigorous empirical testing expected of scientific discipline.

Professor Shunkichi Matsumoto of Tok University, Japan, explores the role of the discipline.

According to defenders of adaptive though a daptive thinking may be methodologically incomplete, it nonetheless works well in reducing the hypothesis space. Evolutionary psychology using adaptive thinking is not doomed as some critics wish to argue it is, as they are in fact only arguing against the discovery of hypotheses, and not their justification. In other words, all that really matters as to whether adaptive thinking is a good heuristic is the truthfulness of the hypotheses after the final testing; the method of obtaining them need not be of any overbearing importance. confirmation, always gets the last wor.

- confing always gets the last word.

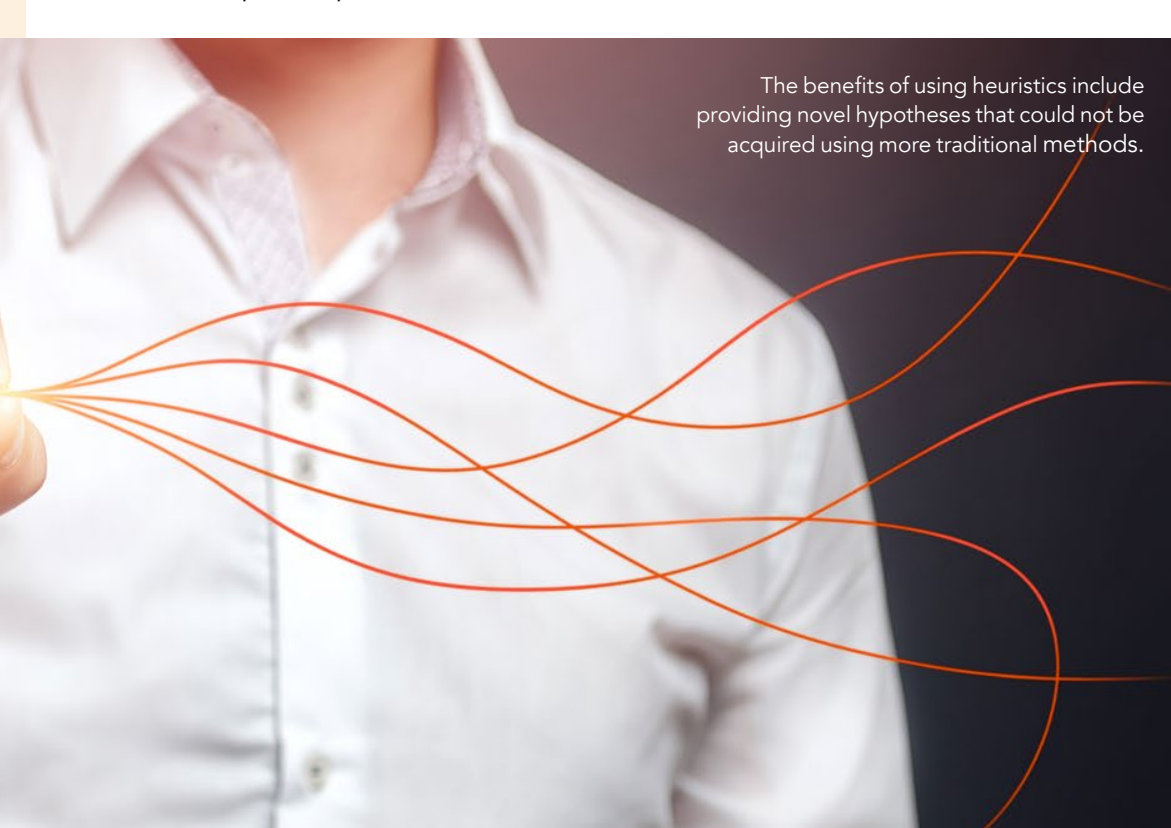

Using relievelof neuristics should be considevodi to ess important in evolutioncy psychology than the conclusivenisfs of the testing orocedure; nature does not always get the last word in estaolishing scientific facts.

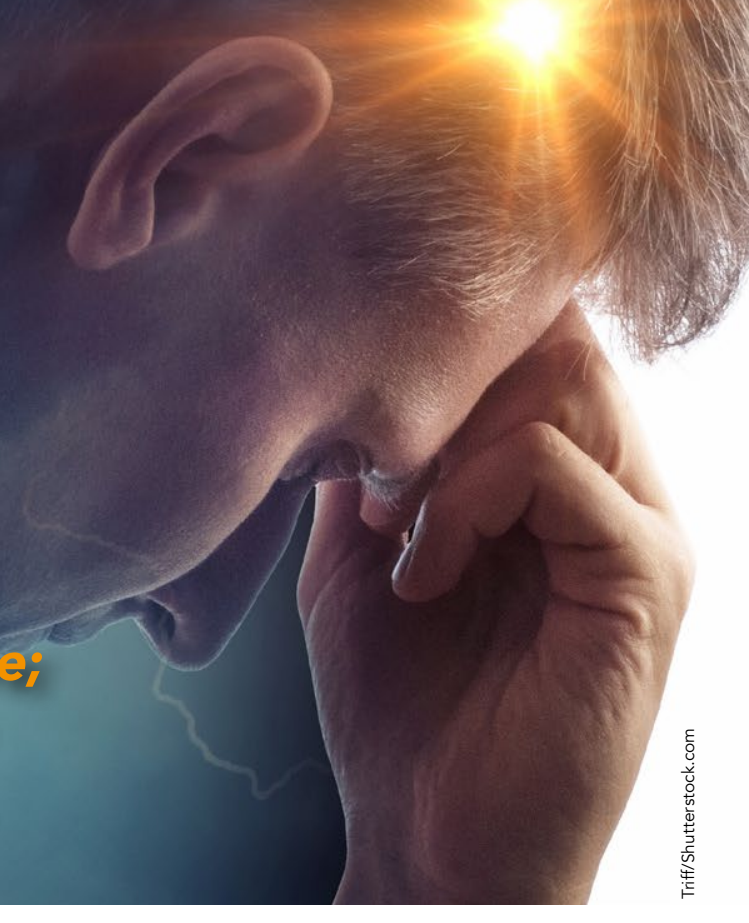

HEURISTICS AND

ADAPTIVE THINKING IN

Firstly, we should further clarify wh the

two key terms of Matsumoto's research

mean. A heuristic is a strategy used in

scientific research to solve problems and

discover hypotheses. It is a pragmatic

method that, while not being perfect or

complete, nonetheless provides sufficien

approximate results that can later be

put under traditional scientific testing.

The benefits of using heuristics include providing novel hypotheses worthy of

attention that could not be acquired

using more traditional methods, as well as being quicker at finding solutions. The based on the fact that they will be further scrutinised once the subsequent resear has been undertaken.

Adaptive thinking is a metho of inferring the structures or behaviours of an evolution from an understanding of 'prespecified selection

pressures' (eg,

the ancestral

landscape

and habitual conditions of the given organism)
As Matsumoto claims, it is a forward looking inference, from past functions (ie, survival traits) to current forms (modern traits). In a way, adaptive thinking is naturally heuristic in value, as it leads to the discovery of previously unknown behavioural features and patterns based on the somewhat ambiguous data of ancestral conditions. In this sense, it can be a good heuristic a it narrows down the amount of potentially true theories from the seemingly infinite number of possible ones, based on

MACHERY AND GOLDFINGH: TWO HEURISTIC (x) or uncover some new
psychological traits, psychological traits,
thereby backing up thereby backing up
the former (backward) inference by the latter (forward) one, and Adaptive thinking leads to the discovery of previously
unknown behavioural features

psychology. One such criticism is of which behavioural trait may have been researchers are not completely ignorant trait and can thus forge an evolutionary features of the trait, as opposed to finding them new and undiscovered. Here, the earch is compromised as it is based on circular reasoning. ing

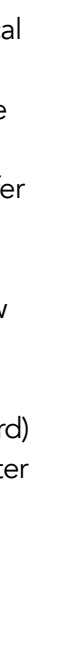

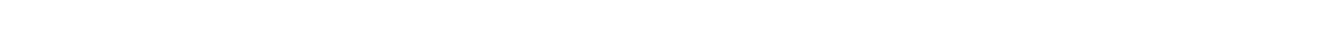


application of the latter strategy. Rather than creating novel research ideas each time evolutionary psychologists method makes it possible that ach new research model would share the same initial basic assumptions with one another and thus, cover up each other's latent biases. As William Wimsat points out, "the errors produced by using a heuristic are not random but systematically biased." Furthermore, if left to researchers from other disciplines to complete, the research may continue to be based on the underlying biases

that the evolutionary psychologist did no manage to notice, given the unlikelihood new on their own.

\section{REQUIREMENTS FOR}

\section{ADAPTIVE THINKING TO}

DE A GOOD HEURISTIC

Matsumme dras two conclusions in (as opposed to arbitrany) heuristics should be considered no less important in evolutionary psychology than the conclusiveness of the final testing procedure; namely, nature does not always get the last word in establishing providing the discipline with its raison work effectively when it is substantiated previously made, ie, that can be designed independently of knowledge about those initial observations. If this equirement is not met, then the charge

Andrew Goldfinch attempts to break free from the charge of circularity by interpreting evolutionary psychology as a euristic project, and not an explanatory one. He argues that evolutionary psychology should not necessarily be
considered a discipline that issues final explanations of phenomena, but rather on that generates hypotheses to be confirmed or denied later on. Evolutionary psychology as a heuristic project therefore, could stop short of having to justify hypotheses, in addition to providing them. It is because thas been expected of evolutionary psychology to provide final declaration of truth, hat issues and objections of circulanty have been raised against it. If instead, evolutionary psychology were scientific facts. The second, which follows from the first, is that although adaptive thinking is a fair heuristic, $d^{\prime}$ etre of tracing the trait in question back to ts histonical onign, it can only by sufficient historical underpinnings. with Goldfinch's argument Matsumoto argues that explanations and

hypotheses are mutually exchangeable
concepts rrather than dichotomous

confirmed or denied, then necessary research.

Using heuristics in this context is susceptible to the same pitfalls, biases, and errors as reductionism in general, since the former is a special application of the latter strategy.

ones, as Goldfinch attempts to explain they are). In science, any hypotheses are products of trying to explain new phenomena, with their provisional character being emphasised. Similarly, any explanations are open to fallibility More to the point, using heuristics in pitfalls, biases, and errors as reduction and are thus hypothetical in nature. this contex is susceptible to the same
If researchers in the field of evolutionary psychology can live up to these requirements and retain an awareness of the aforementioned methodological issues surrounding the practice, then it may eventually psychology and behaviours based on ultimate, evolutionary causes with circular practice. provide deeper understanding of our

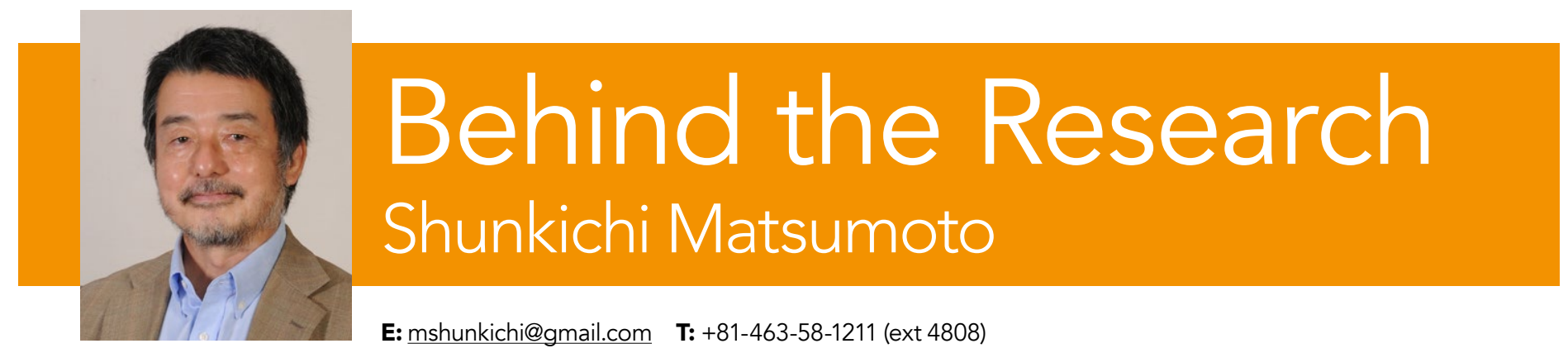

\section{Research Objectives}

Shunkichi Matsumoto undertakes a critical analysis of the methodology of evolutionary psychology.

\section{Detail}

Addres

Sudent Achievement Center

Tokai University

Hiratsuka Kanagawa

Hiratsuka, Kanagawa

Bio

Shunkichi Matsumoto is a professor at the Student Achievement Center at Tokai University in Japan, specialised in the philosophy of biology. He holds bachelor's degree in physics from Kyoto University, a master's degree in HPS from Tokyo University, and a PhD philosophy from Keio University, all in Japan. He has served as an editor-in-chief and now serves as a board member for the Japan Association of Philosophy of Science.

\section{Funding}

Grantin-Aid for Scientific Research (C) from the Ministry Jeducation, Culture, Sports, Science and Technology of apan (Gran Number: JP19K00277).

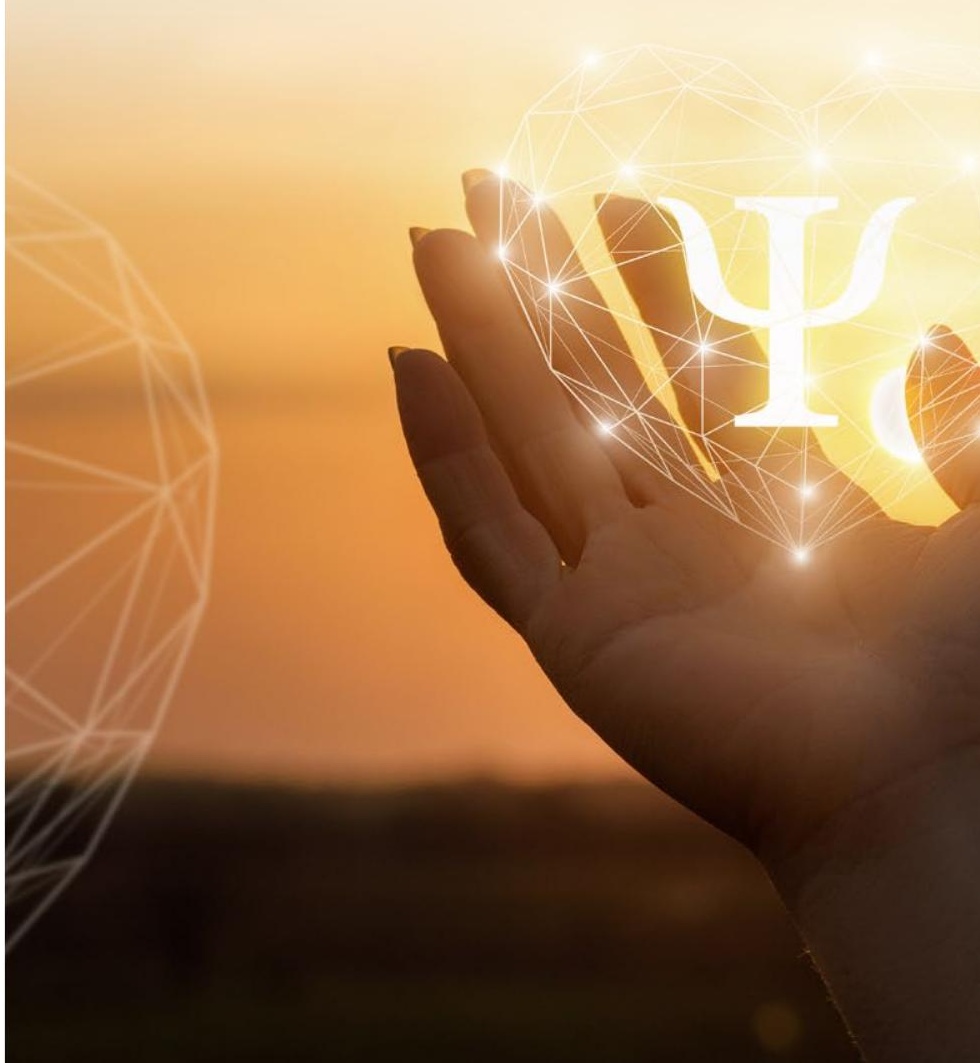

References

Matsumoto, S (2021). Making Sense of the Relationship Between Adaptive Thinking and Heuristics in Evolutionary Psychology. Biological Theory, Vol 16, pp 16-29.

Matsumoto, S (2008). Analyzing 'Evolutionary Functional Analysis' in Evolutionary Psychology. Annals of the Japan

\section{Personal Response}

Can you provide an example of historical underpinnings which substantiate the use of adaptive thinking in

II Many species of migrating birds were known to fly south in winter and fly north in summer. Given this fact, researchers used to appeal to adaptive thinking to the to avoid harsh conditions in winter. However, there remains another adaptive story, of equal plausibility: that those bird were originally of tropical origin and had to migrate north in summer to obtain resources (food or habitat) for breeding; migrating south in winter is just a consequence of migrating

These two incompatible versions of adaptive thinking both sounded plausible. However, it was historical research phylogenetic study of chronological sequence of events cladistic analysis revealed that thour of the latter: the cladistic analyis reveales in those species of bird are

This means that creating plausible hypotheses by adaptive thinking is not sufficient to establish scientific facts: without
historical underpinnings they will remain just-so stories. 